\title{
Selfishness and altruism can coexist when help is subject to diminishing returns
}

\author{
RM Sibly ${ }^{1}$ and RN Curnow ${ }^{2}$ \\ ${ }^{1}$ School of Biological Sciences, University of Reading, Reading, UK and ${ }^{2}$ School of Mathematics and Statistics, University of Reading, \\ Reading, UK
}

\begin{abstract}
Altruism and selfishness are $30-50 \%$ heritable in man in both Western and non-Western populations. This genetically based variation in altruism and selfishness requires explanation. In non-human animals, altruism is generally directed towards relatives, and satisfies the condition known as Hamilton's rule. This nepotistic altruism evolves under natural selection only if the ratio of the benefit of receiving help to the cost of giving it exceeds a value that depends on the relatedness of the individuals involved. Standard analyses assume that the benefit provided by each individual is the same but it is plausible in some cases that as more individuals contribute, help is subject to diminishing returns. We analyse this situation using a single-locus two-allele model of selection in a diploid population with the altruistic allele dominant to the selfish allele. The analysis requires calculation of the relationship between the fitnesses of the
\end{abstract}

genotypes and the frequencies of the genes. The fitnesses vary not only with the genotype of the individual but also with the distribution of phenotypes amongst the sibs of the individual and this depends on the genotypes of his parents. These calculations are not possible by direct fitness or ESS methods but are possible using population genetics. Our analysis shows that diminishing returns change the operation of natural selection and the outcome can now be a stable equilibrium between altruistic and selfish alleles rather than the elimination of one allele or the other. We thus provide a plausible genetic model of kin selection that leads to the stable coexistence in the same population of both altruistic and selfish individuals. This may explain reported genetic variation in altruism in man.

Heredity (2011) 107, 167-173; doi:10.1038/hdy.2011.2; published online 23 February 2011

Keywords: altruism; selfishness; kin selection; polymorphism; heritability

\section{Introduction}

Many families contain a mixture of good and bad, a topic of perennial interest. But can we really have, some of us, both altruistic siblings and selfish siblings? Studies of behaviour genetics show that some $30-50 \%$ of the variation in willingness to help others is heritable (Rushton et al., 1986; Rushton, 2004; Scourfield et al., 2004; Knafo and Plomin, 2006; Hur and Rushton, 2007; Cesarini et al., 2009; Gregory et al., 2009) but cf. (Krueger et al., 2001). This is known from comparison of the altruistic dispositions of monozygotic and dizygotic twins in both Western and non-Western (Hur and Rushton, 2007) populations. Those who do not help others, help themselves, and so anti-social behaviour has a heritability similar to that of prosocial behaviour (Rhee and Waldman, 2002) but cf. (Krueger et al., 2001). So, depending on the genotypes of our parents, my brothers may all be selfish, all altruistic or a mixture of both. The existence of genetically based variation in altruism and selfishness calls for explanation (Rushton, 2004; Rushton et al., 2008). One possibility is that altruism and selfishness have no fitness effects and the observed

Correspondence: Professor RM Sibly, School of Biological Sciences, University of Reading, Whiteknights, Reading RG6 6AS, UK,

E-mail: r.m.sibly@reading.ac.uk

Received 4 November 2010; revised 20 December 2010; accepted 23

December 2010; published online 23 February 2011 polymorphism arose by genetic drift, but we do not regard this as credible. In this study, we show that a mix of altruism and selfishness is an expected outcome of natural selection in a plausible model in which, if several individuals provide help, the fitness benefits to the recipient are subject to a law of diminishing returns.

The modern study of the evolution of altruism began with Hamilton's (Hamilton, 1964) demonstration, that altruistic alleles that cause carriers to help relatives are selected if $b>c / r$ (Hamilton's rule), where $b$ represents a fitness benefit obtained by a relative at a cost $c$ to the helper, and $r$ is the coefficient of relatedness between the relatives. This theory elicited a large quantity of empirical and theoretical work, and the question of how altruistic behaviours evolved through natural selection was until recently considered settled. However, now it is again the subject of debate (Okasha, 2010). The problems stem at root from imprecision in empirical testing of Hamilton's rule. If $b, c$ and $r$ could be measured precisely, then it would be straightforward to check that altruism occurred if and only if Hamilton's rule held. Empirical work has amply confirmed that animals behave more altruistically towards kin than non-kin, and the coefficient of relatedness has often been determined precisely, but estimation of fitness costs and benefits is difficult and has therefore generally been imprecise. Furthermore, only a few studies have attempted quantitatively to evaluate Hamilton's rule in animals. This has left theory with only limited empirical leverage. Theoretical work 
has instead aimed to understand a rich variety of processes that may be at work in the evolution of social behaviour and several approaches have emerged. Early population genetics modelling was comprehensively reviewed by Michod (1982). Subsequently a 'direct fitness' approach was developed using the methods of quantitative genetics (see, for example, Queller, 1985, 1992a, b; Taylor and Frank, 1996; Frank, 1998; Fletcher and Doebeli, 2006), and there are also other strong lines of attack using evolutionary game theory (for example, Sigmund, 1993; Doebeli et al., 2004; Hauert and Doebeli, 2004; Archetti, 2009), and multi-level selection (for example, Nowak et al., 2010). Okasha (2010) considered the relationships between the theoretical approaches to be sometimes ambiguous, and identified disagreement among biologists as to which is most fundamental. However, previous workers have generally agreed that population genetics is the touchstone; other approaches are valid provided they can be translated into population genetics, although with additional assumptions if needed. Recently another powerful line of work studying the evolution of social polymorphisms in bacteria and yeast has emerged (see, for example, Ross-Gillespie et al., 2007; Smith et al., 2010). In these studies, strain-specific strategies were identified ('co-operator', 'cheater') and the fitness of each was measured directly as the number of spores it produced in the next generation. These studies have shown that each strain outperformed the other when at low density, and the fitness differential between the strains changed monotonically with their frequencies. Thus, a stable polymorphism was unequivocally demonstrated empirically. In this and the theoretical approaches described above, fitnesses can be frequency dependent in the sense that the fitness of an individual playing a specified strategy depends on the numbers of individuals playing that and other specified strategies.

However, in none of these approaches does fitness depend on the genotypes of the individual's forebears. Introducing this dependency is the crucial new ingredient in our model. It is needed because in our model fitness depends on the number of encountered relatives who are altruistic, and this is determined by the alleles they carry, which in turn depends on the genotypes of the individual's forebears.

Hamilton (1964) assumed that the benefits of help remain the same as genes causing altruism spread in populations. In reality, however, as more individuals help the benefits each provides may decrease. Suppose I need shelter when injured, food when starving or rescue in battle. The first individual to help may save my life. Latecomers may pay similar costs in attempting to help, but the additional benefit to me is less. There are diminishing returns because there are limits to how much individuals can be helped. Several possibilities are shown in Figure 1a. In the case of constant returns, the benefits per helper are the same irrespective of the number of helpers (case $i$ in Figure 1a) and cumulative benefits increase linearly with the number who help (Figure 2). Cases ii-iv show linear diminishing returns. In this study, we model cases such as these in which help is subject to a law of diminishing returns. Our interest is in investigating when and where genetic polymorphisms may arise in diploid animals as a result of genes with specified effects, which depend on the genotypes of the
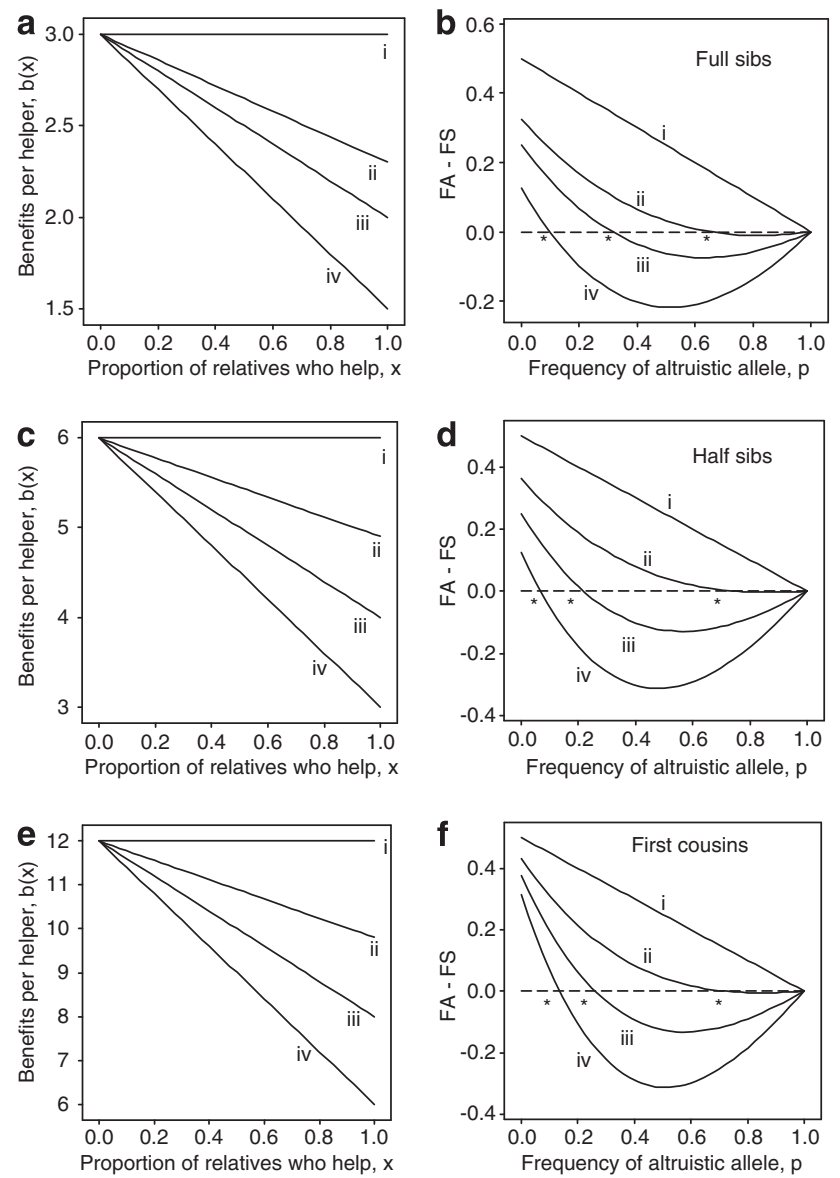

Figure 1 Examples of constant or diminishing returns from helping, and their consequences, for full-sibs (top row), half-sibs (middle row) and first cousins (bottom row). Left-hand figures show benefit per helper in relation to the proportion of relatives who provide help. Benefits are in these diagrams in multiples of the cost of help. In case $i$, the benefits per helper do not vary with the proportion of relatives who provide help, so the cumulative benefits increase linearly with the number of helpers as shown in Figure 2. Cases iiiv show diminishing returns. In each case the pattern of diminishing returns is similar for the different kin relationships (full-sibs, halfsibs and first cousins) once the coefficient of relatedness is taken into account. Thus, $r \cdot b(0)=\frac{1}{2} \times 3=1.5$ for full-sibs, as $r=\frac{1}{2}$ and $b(0)=3$. Similarly $r \cdot b(0)=\frac{1}{4} \times 6=1.5$ for half-sibs, and $1 / 8 \times 12=1.5$ for first cousins. At the other extreme when all relatives help $(x=1)$ the intercept is again similar for the different kin relationships, thus $b(1) / b(0)=0.50$ in case iv, 0.67 in case iii and 0.82 in case ii, except for full-sibs case ii. In this study, we used the value 0.77 because they lack an internal equilibrium if $b(1) / b(0)=0.82$. Right-hand figures: fitness consequences of the possibilities shown in left-hand figures. $F_{\mathrm{A}}-F_{\mathrm{S}}$ represents the fitness differential between the altruistic and selfish alleles, calculated from equations (1)-(3) and here normalised by division by Nc. In case i, the altruistic allele spreads to fixation because for all three relationships, $F_{\mathrm{A}}-F_{\mathrm{S}}>0$, but the fitness differential declines linearly to zero as the frequency of the altruistic allele increases. In cases ii-iv, the altruistic allele increases when rare but reaches a stable equilibrium, marked asterisks $(*)$, where $F_{\mathrm{A}}-F_{\mathrm{S}}=0$.

carrier's sibs. The principal difficulty is in calculating the relationship between the fitnesses of the genotypes and the frequencies of the genes from the phenotypic relationships illustrated in Figures 1a and 2. This is complicated because the genotypes each individual encounters depend on the genotypes of its forebears (as in Table 2). The calculation is simpler without diminishing 


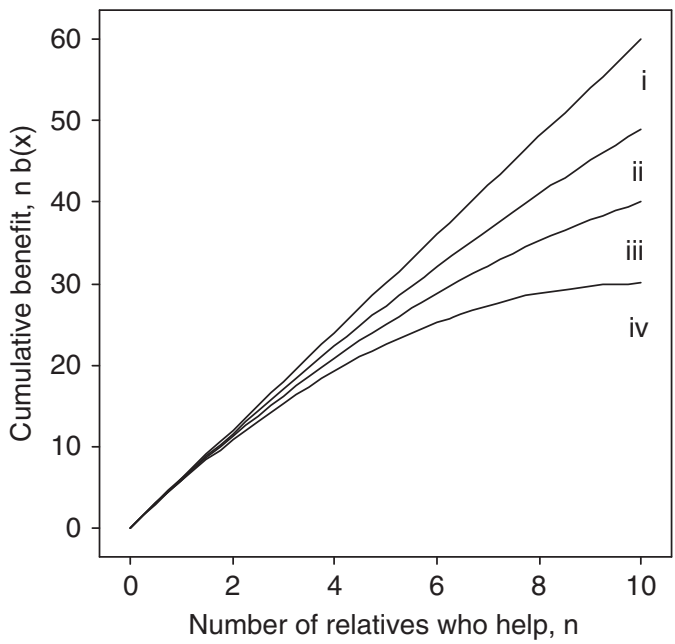

Figure 2 Cumulative benefits shown in relation to the number of relatives, $n$, who help, for cases i-iv of Figure 1a when the sibship size is 10 . The horizontal axis differs from that in Figure 1 because the cumulative benefits, that is, $n \times b(x)$, depend on the number of relatives who help. The number of relatives who help is equal to the sibship size times the proportion of relatives who help.

returns because then the coefficient of relatedness, $r$, is sufficient to determine the frequencies of encounters with different phenotypes within sibships, as Hamilton showed. Without diminishing returns, our results reduce to Hamilton's. With diminishing returns, population genetic models are needed to take account of the diploidy and the way this affects the relative frequencies of genotypes in the carrier's sibs. The calculations are not possible using existing direct fitness or environmental stress screening approaches; these start from a presumption that fitness is a known function of the level of altruism.

No previous population genetic model of altruism has found a condition for a genetic polymorphism other than overdominance, which is discussed by Uyenoyama and Feldman (1981) and Uyenoyama et al. (1981). In this study, we used rigorous population genetic models to investigate the evolutionary consequences of diminishing returns, which we show may lead to the stable coexistence in the same population of both altruistic and selfish individuals.

\section{Population genetics model}

We assume a standard single-locus two-allele model of selection in a diploid population with discrete generations and random mating so that immediately after conception genotypes occur in Hardy-Weinberg proportions. The alleles are labelled $\mathrm{A}$ and $\mathrm{S}$ and their properties are described below. The frequencies of the sib genotypes have to be calculated separately for each possible common ancestor or pair of common ancestors. These frequencies then have to be averaged over the possible common ancestors or pairs of common ancestors. As in the derivation of Hamilton's rule, we have had to assume weak selection so that the genotypic frequencies in previous generations of parents at the time of mating are, despite the effects of altruistic selection, in HardyWeinberg equilibrium. This amounts to ignoring terms of second order in the effects of altruistic selection.
Simulations showed that the effect of this approximation is very small (RM Sibly and RN Curnow, in preparation).

We now calculate the fitness values, $F_{\mathrm{SS}}, F_{\mathrm{SA}}$ and $F_{\mathrm{AA}}$, of the three genotypes that determine the changes in the frequencies of the genotypes as a result of the altruistic selection. The frequency of each genotype after selection is its frequency before selection multiplied by its fitness, normalised by dividing by the sum of (fitness $\times$ frequency before selection) of all three genotypes. The genotypic fitnesses are then used to calculate the fitnesses of the two alleles, $F_{\mathrm{S}}$ and $F_{\mathrm{A}}$. The frequency of the $A$ allele after the altruistic selection is its frequency before selection multiplied by its fitness, normalised by dividing by the average of (fitness $\times$ frequency before selection) of both alleles. The change in the frequency of the A allele because of the altruistic selection is $p q\left(F_{\mathrm{A}}-F_{\mathrm{S}}\right)$ divided by the sum fitness of the two alleles. Thus, the evolution of the two alleles is determined by the sign of $F_{\mathrm{A}}-F_{\mathrm{S}}$. If there are no diminishing returns, the sign of $F_{\mathrm{A}}-F_{\mathrm{S}}$ is given by Hamilton's rule (that is, by the sign of $b / r-c)$.

Let $p$ be the frequency of a dominant 'altruistic' allele, call it $\mathrm{A}$, that causes its carriers to help relatives related by a coefficient of relatedness $r$, in which case each recipient receives a fitness benefit $b(x)$ from each helper, where $x$ is the proportion of relatives who help, but each helper pays a fitness cost $c . b$ will here always be written as a function of the proportion of relatives who help, that is, as $b(x)$; this is not to be confused with the alternative use of brackets to denote grouping in mathematical expressions. Let the alternative allele at the locus be $S$, a recessive non-altruistic allele that occurs with frequency $q=1-p$. Homozygous SS individuals do not help relatives and will be labelled selfish. The net benefits obtained by individuals depend on their phenotypes and on those of their relatives as shown in Table 1.

To calculate the fitness values, we first calculate the proportion of relatives of an individual who are altruistic. This depends on the individual's genotype (SS, SA or AA) and on the genotype(s) of the forebear(s) he shares with his relatives. Using this approach, we derive equations (1)-(3) giving formulae for the difference between the fitnesses $F_{\mathrm{A}}$ and $F_{\mathrm{S}}$ of the altruistic and selfish alleles, respectively, for each of three types of relationship: half-sibs, full-sibs and first cousins, in terms of the frequencies of the two alleles, the values of the benefits and costs, and the size of the sibship. We begin with half-sibs because their mathematical analysis is simpler.

Half-sibs have one parent in common. The genotype of the common parent determines the proportions of offspring that are of each genotype, and these are shown in Table 2a. The average fitness of an offspring of specified genotype is the sum of the product of the frequencies of its encounters with its relatives of each phenotype, times the benefit minus the cost of each encounter. Costs and benefits depend on phenotype as shown in Table 1 . We assume that all pairs of sibs have equal numbers of encounters providing opportunities for help to be received or given. Benefits and costs depend on the proportions of relatives that are selfish or altruistic, and these depend on the genotype of the common parent as specified in Table 2a. Consider offspring of genotype SS with an SS parent. With our assumption that the parental genotypes are in Hardy- 
Table 1 Net benefit to an individual, after the subtraction of costs, of encounters with one of his relatives, classified according to the selfish/altruistic phenotype of each

\begin{tabular}{lcc}
\hline Individual & \multicolumn{2}{c}{ Relative } \\
\cline { 2 - 3 } & Selfish & Altruistic \\
\hline Selfish & $-c$ & $b(x)$ \\
Altruistic & $b(x)-c$ \\
\hline$c$ is the fitness cost of the encounter if the individual is altruistic and \\
$b(x)$ is the fitness benefit the individual obtains from the encounter if \\
a proportion $x$ of his relatives are altruistic.
\end{tabular}

Table 2 Quantities used in fitness calculations

\begin{tabular}{|c|c|c|c|c|c|c|}
\hline \multicolumn{2}{|c|}{ Common forebear(s) } & \multicolumn{5}{|c|}{ Offspring } \\
\hline \multirow[t]{2}{*}{ Genotype(s) } & \multirow[t]{2}{*}{ Frequency } & \multicolumn{3}{|c|}{ Genotype proportions } & \multicolumn{2}{|c|}{ Phenotype proportions } \\
\hline & & SS & $S A$ & $A A$ & Selfish & Altruistic \\
\hline \multicolumn{7}{|l|}{ (a) } \\
\hline SS & $q^{2}$ & $q$ & $p$ & 0 & $q$ & $p$ \\
\hline SA & $2 p q$ & $\frac{1}{2} q$ & $\frac{1}{2}$ & $\frac{1}{2} p$ & $\frac{1}{2} q$ & $\frac{1}{2}+\frac{1}{2} p$ \\
\hline AA & $p^{2}$ & 0 & $q$ & $p$ & 0 & 1 \\
\hline \multicolumn{7}{|l|}{ (b) } \\
\hline $\mathrm{SS} \times \mathrm{SS}$ & $q^{4}$ & 1 & 0 & 0 & 1 & 0 \\
\hline $\mathrm{SS} \times \mathrm{SA}$ & $4 q^{3} p$ & $\frac{1}{2}$ & $\frac{1}{2}$ & 0 & $\frac{1}{2}$ & $\frac{1}{2}$ \\
\hline $\mathrm{SS} \times \mathrm{AA}$ & $2 q^{2} p^{2}$ & 0 & 1 & 0 & 0 & 1 \\
\hline $\mathrm{SA} \times \mathrm{SA}$ & $4 q^{2} p^{2}$ & $\frac{1}{4}$ & $\frac{1}{2}$ & $\frac{1}{4}$ & $\frac{1}{4}$ & $\frac{3}{4}$ \\
\hline $\mathrm{SA} \times \mathrm{AA}$ & $4 p^{3} q$ & 0 & $\frac{1}{2}$ & $\begin{array}{l}\frac{4}{2} \\
\frac{1}{2}\end{array}$ & $0^{4}$ & 1 \\
\hline $\mathrm{AA} \times \mathrm{AA}$ & $p^{4}$ & 0 & $0^{2}$ & 1 & 0 & 1 \\
\hline \multicolumn{7}{|l|}{ (c) } \\
\hline $\mathrm{SS} \times \mathrm{SS}$ & $q^{4}$ & $q$ & $p$ & 0 & $q$ & $p$ \\
\hline $\mathrm{SS} \times \mathrm{SA}$ & $4 q^{3} p$ & $\frac{3}{4} q$ & $\frac{1}{4} q+\frac{3}{4} p$ & $\frac{1}{4} p$ & $\frac{3}{4} q$ & $p+\frac{1}{4} q$ \\
\hline $\mathrm{SS} \times \mathrm{AA}$ & $2 q^{2} p^{2}$ & $\frac{1}{2} q$ & $\frac{1}{2}$ & $\frac{1}{2} p$ & $\frac{1}{2} q$ & $\frac{1}{2}+\frac{1}{2} p$ \\
\hline $\mathrm{SA} \times \mathrm{SA}$ & $4 q^{2} p^{2}$ & $\frac{1}{2} q$ & $\frac{1}{2}$ & $\frac{1}{2} p$ & $\frac{1}{2} q$ & $\frac{1}{2}+\frac{1}{2} p$ \\
\hline $\mathrm{SA} \times \mathrm{AA}$ & $4 p^{3} q$ & $\frac{1}{4} q$ & $\frac{1}{4} p+\frac{3}{4} q$ & $\frac{3}{4} p$ & $\frac{1}{4} q$ & $p+\frac{3}{4} q$ \\
\hline $\mathrm{AA} \times \mathrm{AA}$ & $p^{4}$ & 0 & $q$ & $p$ & 0 & 1 \\
\hline
\end{tabular}

(a) Half-sibs, who share a common parent; (b) full-sibs, who have both parents in common, and (c) first cousins, who share one pair of grandparents. Parents and grandparents collectively are here referred to as forebears, and their possible genotypes are shown in the first two columns together with their frequencies. Remaining columns show, for each forebear genotype, the proportions of offspring that are of each genotype and phenotype. The frequencies of the A and $\mathrm{S}$ alleles are $p$ and $q$ respectively, $p+q=1$.

Weinberg equilibrium, the frequency of these offspring is $q^{2} \cdot q=q^{3}$ from Table 2a. A proportion $q$ of their half-sibs sharing the SS parent are selfish and $p$ are altruistic. Encounters with the former are cost and benefit free, encounters with the latter yield benefit $b(p)$ to the SS offspring. So the net fitness benefit of these encounters is $q^{3}(q \cdot 0+p \cdot b(p))=q^{3} p b(p)$ multiplied by the size of the sibship, $N$, which is assumed constant. This gives the first term on the right-hand side of the equation below. Note that sibship size appears in all cost and benefit terms. Extending the method to the other two types of sibship (second and third terms on the right-hand side of the equation below), we can now obtain the average fitness, $F_{\mathrm{SS}}$ of an $\mathrm{SS}$ individual from the equation

$$
\begin{aligned}
q^{2}\left(F_{\mathrm{SS}}-1\right) & =p q^{3} b(p) N \\
& +p q^{2}(1 / 2 q \cdot 0+(1 / 2+1 / 2 p) b(1 / 2+1 / 2 p)) N \\
& +p^{2} \cdot 0 \cdot N
\end{aligned}
$$

The right-hand side of this equation, derived above, shows the total of the changes to the fitness of the SS genotype that result from encounters between relatives. To obtain the left-hand side note that these changes accrue to the $q^{2}$ copies of the SS genotype (both sides of the equation have been normalised by dividing by population size) and result in an increase in their average fitness above its baseline value of 1 .

Rearranging the equation we have

$$
F_{\mathrm{SS}}=1+p q b(p) N+p(1 / 2+1 / 2 p) b(1 / 2+1 / 2 p) N
$$

\section{Similarly}

$$
\begin{aligned}
& F_{\mathrm{AS}}=1+1 / 2 p q b(p) N+1 / 2(1 / 2+1 / 2 p) b(1 / 2+1 / 2 p) N \\
& \quad+1 / 2 p b(1) N-c N \\
& F_{\mathrm{AA}}=1+q(1 / 2+1 / 2 p) b(1 / 2+1 / 2 p) N+p b(1) N-c N
\end{aligned}
$$

Finally, the fitness of each allele is the average fitness of the genotypes containing the allele, so $F_{\mathrm{A}}-F_{\mathrm{S}}=\left(q F_{\mathrm{AS}}+p\right.$ $\left.F_{\mathrm{AA}}\right)-\left(q F_{\mathrm{SS}}+p F_{\mathrm{AS}}\right)$. This gives

$$
\begin{aligned}
F_{\mathrm{A}}-F_{\mathrm{S}}= & -c q N+1 / 2 p b(1) N-1 / 2 p q b(p) N \\
& +1 / 4(1+p)(q-p) b(1 / 2+1 / 2 p) N
\end{aligned}
$$

Equation (1) provides a formula for calculating the difference between the fitnesses $F_{\mathrm{A}}$ and $F_{\mathrm{S}}$ of the altruistic and selfish alleles in half sibs. We now use the same method to derive equivalent formulae for full sibs and first cousins.

Full-sibs have two parents in common, and there are six possible combinations of parental genotypes. The proportions of offspring of each genotype are shown for each of the six possible parental matings in Table $2 b$. The procedure used for half-sibs here gives

$$
\begin{aligned}
F_{\mathrm{A}}-F_{\mathrm{S}}= & -1 / 2(4 p-1) q^{2} b(1 / 2) N-3 / 2 p(2 p-1) q b(3 / 4) N \\
& +p(1+p) q b(1) N-q c N
\end{aligned}
$$

First cousins have one pair of grandparents in common, and there are six possible combinations of these grandparental genotypes. The proportions of their grandchildren that are of each genotype are shown for each of the six possible grandparental matings in Table $2 \mathrm{c}$ in the 'offspring' columns. The genotypic frequencies in this case are derived by multiplying the frequencies of the alleles in the common grandparents by the frequencies, $p$ and $q$, of the random alleles from the unshared grandparents. The same procedure as before here gives

$$
\begin{aligned}
F_{\mathrm{A}}-F_{\mathrm{S}}= & -1 / 2 p q^{3} b(p) N \\
& +1 / 8(1-4 p)(1+3 p) q^{2} b(p+1 / 4 q) N \\
& +3 / 4 p\left(1-p^{2}\right)(1-2 p) b(1 / 2+1 / 2 p) N \\
& +1 / 8 p^{2}(3-4 p)(p+3) b(p+3 / 4 q) N \\
& +1 / 2 p^{3} b(1) N-q c N
\end{aligned}
$$

\section{Discussion}

Equations (1)-(3) give formulae for the difference between the fitnesses $F_{\mathrm{A}}$ and $F_{\mathrm{S}}$ of the altruistic and selfish alleles, that is, for the relative fitness, $F_{\mathrm{A}}-F_{\mathrm{S}}$, of an altruistic allele. These equations show how the relative fitness of an altruistic allele depends on the frequencies of the two alleles, $p$ and $q=1-p$, the values of the benefits and costs, $b(x)$ and $c$, and sibship size, N. For given 
values of $c, b(x)$ and $N$, the equations show how the relative fitness of an altruistic allele varies with its frequency $p$ in half-sibs, full-sibs and first cousins, respectively. If $F_{\mathrm{A}}-F_{\mathrm{S}}>0$, the $\mathrm{A}$ allele is selected and increases in frequency, whereas the $S$ allele declines. Conversely if $F_{\mathrm{A}}-F_{\mathrm{S}}<0, \mathrm{~S}$ allele is selected. Thus, a new mutation from an $S$ to an $A$ allele will increase in frequency if $F_{\mathrm{A}}-F_{\mathrm{S}}>0$ at $p=0$. Subsequently there are two possibilities:

(i) The $\mathrm{A}$ allele spreads to fixation at $p=1$ if $F_{\mathrm{A}}-F_{\mathrm{S}}$ continues to be positive as $p$ increases. The selfish allele is then eliminated. Note that when there is no diminution in returns equations (1)-(3), all reduce to Hamilton's rule (Hamilton, 1964) that the A allele invades the population only if $b / c>1 / r$, where $r$ is the coefficient of relatedness. To see this, note that if $b(x)=b(0)$, equation (1) simplifies to $F_{\mathrm{A}}-F_{\mathrm{S}}=N q$ $(-c+b / 4)$. So $F_{\mathrm{A}}-F_{\mathrm{S}}>0$ if and only if $b / 4>c$, and this is Hamilton's rule for half-sibs. The other cases are obtained similarly.

An example of constant returns is illustrated in Figure 1, as case $\mathrm{i}$ for each type of relationship. Because returns are constant, $b(x)=b(0)$, and values of $b(0)$ were chosen so that $r b(0)=1.5 c$ for each type of relationship. Thus, Hamilton's rule $b / c>1 / r$ holds for all values of $p$ (see Figure 1 caption), and this is why the $A$ allele is selected, that is, $F_{A}-F_{S}>0$ for all values of $p$, for case $\mathrm{i}$ in the panels on the right-hand side of Figure 1.

(ii) Alternatively, as $p$ increases there comes a point beyond which $F_{\mathrm{A}}-F_{\mathrm{S}}$ is negative. The $\mathrm{A}$ allele is then selected against. In between the low frequencies where $\mathrm{A}$ is selected and the high frequencies where it is selected against, there is a stable equilibrium where $F_{\mathrm{A}}-F_{\mathrm{S}}=0$. At the stable equilibrium the population contains both selfish and altruistic individuals. Examples of stable equilibria are given for each type of relationship on the right-hand side of Figure 1 (cases ii-iv). The positions of the equilibria are marked by asterisks. The equilibrium frequencies of the A allele and the corresponding proportions of altruistic individuals are given for each of the three types of sibship in Table 3 . Note that as the lines relating $b(x)$ to $x$ get steeper (case i through iv) the stable equilibria occur at lower values of $p$, and result in higher frequencies of selfish individuals.

The existence of stable equilibria can be understood intuitively using Hamilton's rule. Altruists are selected when there are few helpers because there is then little diminution of returns from helping, so Hamilton's rule holds provided $b(0)>c / r$. At the other extreme altruists are abundant and diminution of returns is maximal, so the benefits obtained per individual are reduced. With sufficient diminution, Hamilton's rule no longer holds and selfish individuals are at an advantage. In sum, altruists are selected when rare but selected against when common. The result is an evolutionary equilibrium with some individuals selfish and others altruistic.

Our results reduce to Hamilton's rule if the returns from helping are constant (no diminishing returns). With diminishing returns, our method shows what happens for the realistic case that fitness depends on the number of encountered relatives who are altruistic. Whether a relative is altruistic depends on his genotype, and that depends on the genotypes of his immediate forebears. Without these dependencies a simpler analysis would be feasible. Fitness would then depend only on the coefficient of relatedness and the level of overall altruism in the population, so direct fitness or environmental stress screening models could be used.

The benefit functions $b(x)$ illustrated in Figure 1 are linear, but this is not assumed in deriving equations (1) (3). The equations can be used whatever form is taken by the $b(x)$ function and stable equilibria may again occur. For full-sibs, for example, the conditions on $b(1 / 2)$, $b(3 / 4)$ and $b(1)$ in (2) that lead to $F_{\mathrm{A}}-F_{\mathrm{S}}=0$ for gene frequencies between 0 and 1 could be used to show the extent of nonlinearity that results in stable equilibria. In deriving equations (1)-(3), we assumed each individual had $N$ relatives and encountered them once each. Changing the frequency of encounters, or making them probabilistic, could be incorporated by modifying the definition of $N$. Our hope is that empiricists will use equations (1)-(3) and in particular will attempt to quantify costs and benefits. To calculate costs and benefits in practice one would use Charlesworth's method for calculating genotype fitnesses for iteraparous life histories (Charlesworth, 1994). Fitness of genotype $i$ is then defined as the number, $F_{i}$ which solves the Euler-Lotka equation $1=\frac{1}{2} \sum F_{i}^{x} l_{i}(x) n_{i}(x) \ldots$ where $l_{i}(x)$ and $n_{i}(x)$ represent respectively survival from birth to age $x$, and expected number of offspring then produced, by individuals of genotype $i$. As an example, help costs 0.007 in fitness units that involves young adults taking a $20 \%$ risk of dying, if one assumes their genotype reaches adulthood at age 15 years with chance 0.4 , and one baby is then born every four years if the adult survives, which happens with chance 0.95 per year (see Sibly (2002) for calculation details).

We hope our analysis will spur further theoretical investigations and also experimentation to estimate the

Table 3 Equilibrium values of the frequencies of the altruistic allele

\begin{tabular}{|c|c|c|c|c|c|c|}
\hline \multirow[t]{2}{*}{ Diminishing returns } & \multicolumn{2}{|c|}{ Full-sibs } & \multicolumn{2}{|c|}{ Half-sibs } & \multicolumn{2}{|c|}{ First cousins } \\
\hline & Frequency & Proportion & Frequency & Proportion & Frequency & Proportion \\
\hline i & 1 & 1 & 1 & 1 & 1 & 1 \\
\hline ii & 0.65 & 0.88 & 0.76 & 0.94 & 0.73 & 0.92 \\
\hline iii & 0.31 & 0.53 & 0.22 & 0.39 & 0.26 & 0.46 \\
\hline iv & 0.10 & 0.19 & 0.07 & 0.13 & 0.14 & 0.25 \\
\hline
\end{tabular}

These vary between the three types of sibship and the four diminishing return models. Frequency ' $p$ ' of the altruistic allele; proportion of altruistic individuals, $p^{2}+2 p q$. 
benefit and cost parameters associated with equilibria in populations containing both selfish and altruistic individuals. Help comes in many forms, presumably with different genetic bases, for example, alloparental care during child rearing (Hawkes et al., 1997; Hrdy, 2009), provisioning during times of need (Allen-Arave et al., 2008), and defence of resources against competitors (Rose and Marshall, 1996). Each and any of these may be subject to laws of diminishing returns, which may be linear as in Figure 1 or nonlinear. The form of the relationship between help received and number of helpers requires quantitative description. From the quantitative form of the relationship the expected mix of selfishness and altruism can be calculated using equations (1)-(3).

The frequencies of the three genotypes calculated for each set of common forebears are exact only when the sibship sizes are infinite but we have shown, by exact calculations, that equilibria can exist even when an individual has only two relatives. It may also be objected that potential helpers may in some situations adjust the amount of help they provide according to the number of other altruists present, thus reducing the cost of helping. This situation is not represented in our simplistic model. However there are other situations where altruists would not be aware of other altruists, for example, because they could not see them. It may also be that the genetic determination of altruism is polygenic, not single locus. Polygenic determination could be represented in a model for an underlying liability that determines altruism or selfishness according to some threshold value on the liability scale. Conditions under which this leads to stable equilibria need investigation. Accepting the simplifications inherent in our model, our results do provide an explanation for the otherwise puzzling finding that prosocial and antisocial behaviours are heritable (Rushton et al., 1986; Rhee and Waldman, 2002; Rushton, 2004; Scourfield et al., 2004; Knafo and Plomin, 2006; Hur and Rushton, 2007; Cesarini et al., 2009; Gregory et al., 2009).

The existence of innately selfish and innately altruistic individuals provides an opening for the evolution of discriminating individuals, who withhold help from selfish individuals and only help those who help others. The basis for this discrimination could be simple observation of which individuals are altruistic and selfish in social groups, such as those in which great apes live. Such discrimination could involve keeping tally of favours returned, as has been observed in man (Hill and Kaplan, 1993; Gurven, 2006). This would be more direct than a system of discrimination based on kin recognition, which has been much discussed (see, for example, Rousset and Roze, 2007). Further analysis is needed to understand under what conditions and to what extent genes for discrimination enter populations at equilibrium between selfishness and altruism.

\section{Conflict of interest}

The authors declare no conflict of interest.

\section{Acknowledgements}

We thank Mark Pagel, Marco Archetti and two anonymous referees for constructive comments on an earlier draft.

\section{References}

Allen-Arave W, Gurven M, Hill K (2008). Reciprocal altruism, rather than kin selection, maintains nepotistic food transfers on an Ache reservation. Evol Hum Behav 29: 305-318.

Archetti M (2009). Cooperation as a volunteer's dilemma and the strategy of conflict in public goods games. J Evol Biol 22: 2192-2200.

Cesarini D, Dawes CT, Johannesson M, Lichtenstein P, Wallace $B$ (2009). Genetic variation in preferences for giving and risk taking. Q J Econ 124: 809-842.

Charlesworth B (1994). Evolution in Age-structured Populations. Cambridge University Press: Cambridge.

Doebeli M, Hauert C, Killingback T (2004). The evolutionary origin of cooperators and defectors. Science 306: 859-862.

Fletcher JA, Doebeli M (2006). How altruism evolves: assortment and synergy. J Evol Biol 19: 1389-1393.

Frank SA (1998). Foundations of Social Evolution. Princeton University Press: Princeton, NJ.

Gregory AM, Light-Hausermann JH, Rijsdijk F, Eley TC (2009). Behavioral genetic analyses of prosocial behavior in adolescents. Dev Sci 12: 165-174.

Gurven M (2006). The evolution of contingent cooperation. Curr Anthropol 47: 185-192.

Hamilton WD (1964). The genetical evolution of social behaviour (I \& II). J Theor Biol 12: 1-16; 17-52.

Hauert C, Doebeli M (2004). Spatial structure often inhibits the evolution of cooperation in the snowdrift game. Nature 428: 643-646.

Hawkes K, Oconnell JF, Jones NGB (1997). Hadza women's time allocation, offspring provisioning, and the evolution of long postmenopausal life spans. Curr Anthropol 38: 551-577.

Hill K, Kaplan H (1993). On why male foragers hunt and share food. Curr Anthropol 28: 701-710.

Hrdy SB (2009). Mothers and Others: The Evolutionary Origins of Mutual Understanding. Harvard University Press: Cambridge, MA.

Hur YM, Rushton JP (2007). Genetic and environmental contributions to prosocial behaviour in 2-to 9-year-old South Korean twins. Biol Lett 3: 664-666.

Knafo A, Plomin R (2006). Prosocial behavior from early to middle childhood: genetic and environmental influences on stability and change. Dev Psychol 42: 771-786.

Krueger RF, Hicks BM, McGue M (2001). Altruism and antisocial behavior: independent tendencies, unique personality correlates, distinct etiologies. Psychol Sci 12: 397-402.

Michod RE (1982). The theory of kin selection. Ann Rev Ecol Syst 13: 23-55.

Nowak MA, Tarnita CE, Wilson EO (2010). The evolution of eusociality. Nature 466: 1057-1062.

Okasha S (2010). Altruism researchers must cooperate. Nature 467: 653-655.

Queller DC (1985). Kinship, reciprocity and synergism in the evolution of social behaviour. Nature 318: 366-367.

Queller DC (1992a). Quantitative genetics, inclusive fitness, and group selection. Am Nat 139: 540-558.

Queller DC (1992b). A general-model for kin selection. Evolution 46: 376-380.

Rhee SH, Waldman ID (2002). Genetic and environmental influences on antisocial behavior: a meta-analysis of twin and adoption studies. Psychol Bull 128: 490-529.

Rose L, Marshall F (1996). Meat eating, hominid sociality, and home bases revisited. Curr Anthropol 37: 307-338.

Ross-Gillespie A, Gardner A, West SA, Griffin AS (2007). Frequency dependence and cooperation: theory and a test with bacteria. Am Nat 170: 331-342.

Rousset F, Roze D (2007). Constraints on the origin and maintenance of genetic kin recognition. Evolution 61: 2320-2330.

Rushton JP (2004). Genetic and environmental contributions to pro-social attitudes: a twin study of social responsibility. Proc R Soc B 271: 2583-2585. 
Rushton JP, Bons TA, Hur YM (2008). The genetics and evolution of the general factor of personality. J Res Pers 42: 1173-1185.

Rushton JP, Fulker DW, Neale MC, Nias DKB, Eysenck H] (1986). Altruism and aggression-the heritability of individual differences. J Pers Soc Psychol 50: 1192-1198.

Scourfield J, John B, Martin N, McGuffin P (2004). The development of prosocial behaviour in children and adolescents: a twin study. J Child Psychol Psychiatry 45: 927-935.

Sibly RM (2002). Life history theory. In: Pagel M. (ed). Encyclopedia of Evolution. Oxford University Press: Oxford, pp 623-627.
Sigmund K 1993. Games of Life. Oxford University Press: Oxford. Smith J, Van Dyken JD, Zee PC (2010). A generalization of Hamilton's rule for the evolution of microbial cooperation. Science 328: 1700-1703.

Taylor PD, Frank SA (1996). How to make a kin selection model. J Theor Biol 180: 27-37.

Uyenoyama MK, Feldman MW (1981). On relatedness and adaptive topography in kin selection. Theor Popul Biol 19: $87-123$.

Uyenoyama MK, Feldman MW, Mueller LD (1981). Population genetic theory of kin selection-multiple alleles at one locus. Proc Natl Acad Sci USA Biol Sci 78: 5036-5040. 